\title{
Cognitive Impairment in Patients with a SCHIZOAFFECTIVE DisORDER: A Comparison with Bipolar Patients in Euthymia
}

\author{
G. Studentkowski1 , D. Scheele ${ }^{1}$, P. Calabrese², F. Balkau ${ }^{1}$, J. Höffler ${ }^{3}$, T. Aubel ${ }^{4}$, M.-A. Edel ${ }^{1}$, G. Juckel ${ }^{1}$, \\ H.-J. Assion ${ }^{5}$ \\ ${ }^{1}$ Department of Psychiatry and Psychotherapy, LWL Hospital, Ruhr-University Bochum, Germany; \\ ${ }^{2}$ Department of Psychology, University of Basel, Switzerland, \\ ${ }^{3}$ Department of Psychiatry and Psychotherapy, Martin-Luther Hospital, Bochum, Germany, \\ ${ }^{4}$ Department of Psychiatry and Psychotherapy, LWL Hospital, Dortmund, Germany, \\ ${ }^{5}$ Department of Psychiatry and Psychotherapy, gpz, Detmold, Germany
}

\begin{abstract}
Objectives: Several studies have shown persistent neurocognitive impairment in patients with a bipolar affective disorder (BD) even in euthymia as well as in patients with a schizoaffective disorder (SAD). The aim of our study was to compare the neuropsychological performance between these two groups. Confounding variables were controlled to enhance our understanding of cognitive dysfunction in both BD and SAD.

Methods: Several domains of neurocognitive function, executive function, memory, attention, concentration and perceptuomotor function were examined in $28 \mathrm{eu}-$ thymic SAD patients and $32 \mathrm{BD}$ patients by using a neuropsychological test battery. The Hamilton Depression Rating Scale (HAMD), Montgomery-Asberg Depression Rating Scale (MADRS) and Young Mania Rating Scale (YMRS) were used to evaluate the patients' clinical status. Data analysis was performed by using a multivariate analysis of covariance (ANCOVA/MANCOVA).

Results: Euthymic SAD patients showed greater cognitive impairment than euthymic BD patients in the tested domains including declarative memory and attention. Putative significant group differences concerning cognitive flexibility vanished when controlled for demographic and clinical variables. Age and medication were robust predictors to cognitive performance of both SAD and BD patients.

Conclusions: Our results point out the worse cognitive outcome of SAD compared to BD patients in remission. Remarkably, the variance is higher for some of the test results between the groups than within each group, this being discussed in light of the contradictive concept of SAD.
\end{abstract}

Key words: bipolar disorder, schizoaffective disorder, cognition, neuropsychology

\section{INTRODUCTION}

It has been estimated that between $0.5 \%$ and $0.8 \%$ of the general population suffer from a schizoaffective disorder (SAD) [3]. Despite slightly different concepts of the DSM-IV and ICD-10 diagnostic criteria, the core feature of SAD in both manuals is the concurrent or consecutive occurrence of psychotic and affective symptomatology. The ongoing debate whether SAD does really exist or is rather an artificial or intermediate category, particularly arises from the differing SAD diagnostic criteria. In a five-years retrospective study of 61 patients with initial diagnosis of SAD a proportion of 37 patients shifted to the diagnosis of bipolar disorder (BD) during the observational period [36]. Consequently, Marneros and Goodwin [30] suggested an adaptation of the ICD-10 classification and to subsume SAD to the affective category rather than to the spectrum of schizophrenia (SZ).

It is further known that cognitive impairment is a characteristic feature of SAD and considered to be a core symptom of both BD and SZ. Cognitive deficits are neither exclusively a result of the affective symptomatology nor solely a consequence of pharmacological treatment [20].

Most of the studies concerning cognitive functioning have shown cognitive deficits in bipolar depression. In recent years, persistent cognitive deficits have been reported even in euthymia in about one third of bipolar patients [19]. Patients with BD exhibit a reduced verbal [51] and spatial working memory [16]. Even when controlled for age, premorbid IQ or residual depressive symptomalogy, the performance is poorer concerning learning, short and delayed recall as well as recognition tasks [32]. The verbal recovery of information best predicts the outcome of psychosocial functioning of bipolar patients, and consequently correlates with serious occupational or relational problems [31]. However, Clark, Iversen and Goodwin [9] also controlled for mild affective symptoms and found a reduced sustained attention, only. A reduced selective attention was evaluated using the Stroop Word Colour Test [50]. Moreover, severe deficits in executive dysfunction were observed in many studies. A prolonged planning time [40], reduced verbal fluency [22] and impaired working memory [46] were the main cognitive domains found in these studies. The result was that the cognitive flexibility was also impaired, and appeared to be distinctive for bipolar patients with 
psychotic features [1]. Despite these convincing data, cognitive performance is significantly poorer in many, but not all executive tasks, Thompson et al. [47] characterized the patient's executive deficit as a generic deficit in controlled processing. A meta-analytic review [38] about cognitive deficits in euthymic BD patients analysed the largest effect size $(d \geq 0.8)$ for aspects of executive function and verbal learning. On the one hand, bipolar patients were partly aware of these cognitive deficits, on the other, they also showed poorer neuropsychological performance without subjective complaints [33]. Nevertheless, there are BD patients with no neuropsychological deficits. According to recent data approximately half of the patients performed without significant difference to healthy controls [3]. Patients with SZ also suffer from widespread neuropsychological deficits, including speed of processing, attention, working memory, verbal and visual learning as well as reasoning, problem solving and social cognition [20]. The impairment is considered to be more severe in SZ than in BD [25] and is not attenuated when statistically controlled for IQ, which was significantly lower in patients with SZ [43]. Burdick et al. [7] found the neurocognitive performance to be longitudinally more stable in SZ than in BD. The latter showed better symptom remission in a 5-years followup, especially in short-term and long-term memory.

Despite the great plenty of studies comparing BD with SZ [11, 18, 34], only a few studies distinctly compared cognitive deficits of $\mathrm{BD}$ patients with SAD patients. Torrent et al. [48] found significant impairment in verbal learning and memory in SAD patients. Glahn et al. [17] reported that SAD patients neither significantly differ from BD patients nor from SZ patients in measures of verbal working memory (spatial delayed response task, forward and backward digit span). Szoke et al. [45] concluded that on the one hand SAD patients closely resemble SZ patients in some of the cognitive domains, on the other hand cognitive deficits are ranging on a continuum from SZ to $\mathrm{BD}$.

It is relevant to mention the negative effect of psychotropic medication on cognition. It is well known, that psychotropic drugs with an antidopaminergic profile (e.g. D2/D3 antagonists) induce on one hand a mesocortical hypodopaminergic state, that is correlated with negative symptoms and cognitive impairment, and on the other hand mesolimbic hyporeactivity that is associated with depressive symptoms.

The aim of our study was to contribute data of cognitive functioning of SAD and BD patients in euthymia further and to evaluate the effect sizes of confounding variables on neuropsycho-logical impairment for both disorders.

\section{Patients And Methods}

\section{SUBJECTS}

The study was conducted at the Department of Psychiatry and Psychotherapy, Ruhr University of Bochum, Germany. Thirty-two Bipolar I and twentyeight patients with Schizoaffective disorder of either gender aged between 18 and 75 years were recruited. The wide age range was intended to allow an analysis of a subsample with elder age.
All were euthymic and met the DSM-IV criteria of bipolar or schizoaffective disorder. Diagnoses were confirmed using the Structured Clinical Interview for DSM-IV (SCID). Subjects with disorders that could be related to neuropsychological impairment (e.g. any significant neurological or medical condition, for instance cardiovascular or pulmonary disease, history of head injury, neurodegenerative disorder, mental retardation, substance dependence within the past year, electroconvulsive therapy in the past 6 months, treatment with benzodiazepines) were excluded from the study. No structural brain abnormalities were reported from the history of the patients or from neuroimaging scans.

After clinical remission for at least 3 months, patients were screened for euthymia on the day of neuropsychological testing using Hamilton Depression Rating Scale (HAMD) or Montgomery-Asberg Depression rating Scale (MADRS) and Young Mania Rating Scale (YMRS). All of the raters attended a ratertraining and the interrater agreement was substantial $(\kappa=0.71)$. HAMD scores had to be less than 8 points each, MADRS scores were lower than 12 points. Patients of the SAD sample did not completly fulfill the remission criteria.

With respect to medication patients in the SAD group were less often on a monotherapy with antipsychotics, but did not differ to the BD group concerning combination therapy or the proportion of compounds with D2/D3 receptor antagonistic properties.

All patients gave written informed consent to participate in the study after the procedures had been fully explained. Ethic approval for the study was granted by the ethics committee of the Ruhr University of Bochum. The study was supported by a grant from the Ruhr University of Bochum, Germany (FoRUM).

\section{Neuropsychological Assessment}

The neuropsychological test battery was composed of multiple well-established and some innovative instruments: MWT-B, d2-test, TMT, subsection of WAIS-R, VLMT and logical memory test.

Estimated premorbid intelligence, MWT-B [26]: In a 37-item task, the patients had to identify one right word out of four artificial words. This measure delivers an estimate of current intellectual function and a clue to premorbid intelligence.

Attention, psychomotor speed and cognitive flexibility, $d 2$ - test [6]: A presentation of 47 letters ( $d$ or $p$ ) with two bars above or under a letter in 14 consecutive rows. Patients have to pass through a row within a time frame of 20 seconds. Parameters are speed (all marked letters, GZ), accurateness $(\mathrm{F} \%)$ and concentration performance (number of correct marks and less confusion mistakes, $\mathrm{KL}$ ).

Trail making test, TMT [27, 35, 37]: Part A is a measure for psychomotor speed, part B is used to measure executive functioning and reflects the ability to shift strategy and visuospatial working memory.

Declarative memory and verbal learning, digit span and block span subtest, subsection of Wechsler Adult Intelligence Scale [49, 21]: Subjects have to repeat a fixed random series of verbal (digit) and visual (block) num- 
bers of increasing length in direct (forward) and reverse (backward) order. Auditory attention, visual attention and short-term retention capacity are assessed in addition to the ability to manipulate the information in the verbal and visual working memory.

Verbal Learning Memory Test, VLMT [23]: The VLMT is an equivalent to the Auditory Verbal Learning Test (AVLT) and comprises a list-learning task of 15 semantic independent words. It contains five immediate free recall trials of a list $\mathrm{A}$, which provides a global measure of learning performance. An interference list $\mathrm{B}$ is presented afterwards and a short-delay recall of list $\mathrm{A}$ in free form is required. After a time span of 20 minutes, list A is repeatedly questioned (long delay free recall).
Logical memory, emotional versus neutral text samples [8]: For resolving the question whether patients benefit from an emotional content of an information in terms of a higher reproduction performance in comparison to a neutral text. Recognition ability of both sixteen item versions is recorded.

\section{STATISTICAL ANALYSES}

Baseline demographic measures and clinical characteristics (Table 1) were compared between bipolar and schizoaffective patients using one-way analyses of variance (ANOVA) and Chi-square tests. Performance on neuropsychological tests was compared by means of multivariate analyses of covariance (MANCOVA).

Table 1. Demographic and clinical characteristics of 32 bipolar and 28 schizoaffective disorder patients.

\begin{tabular}{|c|c|c|c|c|c|c|c|}
\hline \multirow[t]{2}{*}{$\begin{array}{l}\text { Demographic and } \\
\text { clinical variables }\end{array}$} & \multicolumn{2}{|c|}{$\begin{array}{l}\text { Bipolar disorder } \\
\text { patients }(n=32)\end{array}$} & \multicolumn{2}{|c|}{$\begin{array}{l}\text { schizoaffective } \\
\text { patients }(n=28)\end{array}$} & \multicolumn{3}{|c|}{ ANOVA } \\
\hline & Mean & SD & Mean & SD & $F$ & $d f$ & $\mathbf{P}$ \\
\hline Age & 48.0 & 14.92 & 45.61 & 10.5 & 0.5 & $1 ; 58$ & 0.48 \\
\hline YMRS score & 2.34 & 2.67 & 7.63 & 6.37 & 18.29 & $1 ; 57$ & $<0.01$ \\
\hline HAMD score & 4.5 & 2.81 & & & & & \\
\hline MADRS score & & & 10.87 & 8.01 & & & \\
\hline $\begin{array}{l}\text { Years since having a } \\
\text { pychiatric diagnosis }\end{array}$ & 11.56 & 9.61 & 21.39 & 11.34 & 12.04 & $1 ; 53$ & $<0.01$ \\
\hline \multirow[t]{2}{*}{ MWT-B score } & 114.69 & 15.54 & 104.64 & 16.13 & 5.98 & $1 ; 58$ & 0.02 \\
\hline & $\mathbf{n}$ & $\%$ & $\mathrm{n}$ & $\%$ & $\chi^{2}$ & $d f$ & $\mathbf{P}$ \\
\hline \multicolumn{8}{|l|}{ Sex } \\
\hline Male & 13 & 40.6 & 7 & 25 & 1.64 & 1 & 0.2 \\
\hline Female & 19 & 59.4 & 21 & 75 & & & \\
\hline \multicolumn{8}{|l|}{ Education: } \\
\hline No school & 0 & 0 & 2 & 7.7 & 2.55 & 1 & 0.11 \\
\hline Only school & 6 & 18.8 & 5 & 19.2 & 0.002 & 1 & 0.96 \\
\hline $\begin{array}{l}\text { School and futher } \\
\text { education }\end{array}$ & 17 & 53.1 & 14 & 53.8 & 0.003 & 1 & 0.96 \\
\hline Highschool & 9 & 28.1 & 5 & 19.2 & 0.62 & 1 & 0.43 \\
\hline \multicolumn{8}{|l|}{ Current mediacation: } \\
\hline $\mathrm{AD}$ only & 2 & 6.3 & 0 & 0 & 1.75 & 1 & 0.19 \\
\hline MS only & 3 & 9.4 & 7 & 25.9 & 2.85 & 1 & 0.09 \\
\hline NL only & 6 & 18.8 & 0 & 0 & 5.83 & 1 & 0.02 \\
\hline $\mathrm{AD} \& \mathrm{MS}$ & 11 & 34.4 & 3 & 11.1 & 4.38 & 1 & 0.04 \\
\hline $\mathrm{AD} \& \mathrm{NL}$ & 7 & 21.9 & 4 & 14.8 & 0.48 & 1 & 0.49 \\
\hline MS \& NL & 16 & 50 & 16 & 59.3 & 0.51 & 1 & 0.48 \\
\hline All & 7 & 21.9 & 2 & 7.4 & 2.37 & 1 & 0.12 \\
\hline Neither & 1 & 3.1 & 2 & 7.4 & 0.56 & 1 & 0.46 \\
\hline \multicolumn{8}{|l|}{ Last episode: } \\
\hline Maniac & 18 & 56.3 & 16 & 57.1 & 0.01 & 1 & 0.94 \\
\hline Depressiv & 11 & 34.3 & 4 & 14.3 & 3.21 & 1 & 0.07 \\
\hline Mixed & 3 & 9.4 & 5 & 17.9 & 0.93 & 1 & 0.34 \\
\hline
\end{tabular}

ANOVA = Analysis of Variance; YMRS = Young Mania Rating Scale; HAMD = Hamilton Depression Rating Scale; MWT-B = Estimated premorbid intelligence; $\mathrm{AD}=$ Antidepressants; $\mathrm{MS}=\operatorname{mood}$ stabilizer; $\mathrm{NL}=$ Antipsychotics 
All demographic and clinical variables, which were found to be significantly different between these two groups, were included in the analyses as covariates. Consecutively, one-way analyses of covarinace (ANCOVA) were used to detect group differences with single test dimenions. Estimates of effect size were calculated for untransformed data with the formula

$$
\frac{\mu_{\text {bipolar }}-\mu_{\text {schizoaffective }}}{\sigma_{\text {pooled }}}=d[24] \text {. }
$$

Additionally, a stepwise regression analysis was conducted in order to assess the association of clinical and demographic variables with cognitive functioning. Age, gender, years since having a psychiatric diagnosis, hospitalisation rate, number of outpatient treatments, premorbid IQ, YMRS scores, HAMD score resp. MADRS score and current medication were used as variables.

In order to prevent an apha-inflation due to multiple testing, only results with a significance level at
$P<0.01$ were interpreted. Having stated no a-priori hypothesis regarding the direction of differences only two-tailed significance tests were computed. Data analysis was performed using Statistical Packages for Social Sciences (SPSS) version 14.0 [44].

\section{RESULTS}

\section{Demographic Measures and Clinical CHARACTERISTICS}

Sociodemographic and clinical variables are summarized separately for 32 Bipolar I and 28 schizoaffective patients (Table 1). Groups were well matched in terms of age, gender ratio, education and the type of the last episode. The duration of illness differed significantly between the SAD (21,39 years) and BD (11,56 years) group. Gender differences were obvious in both groups with the highest rate of females in the SAD

Table 2. Neuropsychological testing results (estimated means).

\begin{tabular}{|c|c|c|c|c|c|c|c|c|}
\hline \multirow[t]{2}{*}{ Test dimension } & \multicolumn{2}{|c|}{$\begin{array}{l}\text { Bipolar patients } \\
(\mathrm{n}=32)\end{array}$} & \multicolumn{2}{|c|}{$\begin{array}{l}\text { Schizoaffective } \\
\text { patients }(n=28)\end{array}$} & \multicolumn{3}{|c|}{ MANCOVA/ ANCOVA\# } & \multirow[b]{2}{*}{ d } \\
\hline & Mean & SD & Mean & SD & $\mathbf{F}$ & $d f$ & $\mathbf{P}$ & \\
\hline \multicolumn{9}{|l|}{$\begin{array}{l}\text { Attention and } \\
\text { psychomotor speed }\end{array}$} \\
\hline d2 speed (GZ) & 454.60 & 106.56 & 494.81 & 102.24 & $\begin{array}{l}6.94 \\
1.12\end{array}$ & $\begin{array}{l}4 ; 44 \\
1 ; 47\end{array}$ & $\begin{array}{l}<0.01 \\
0.30\end{array}$ & 0.39 \\
\hline $\mathrm{d} 2$ accurateness $(\mathrm{F} \%)$ & 14.36 & 8.32 & 30.50 & 17.52 & 12.37 & $1 ; 47$ & $<0.01$ & 1.18 \\
\hline d 2 concentration $(\mathrm{KL})$ & 149.57 & 58.66 & 140.95 & 63.08 & 0.16 & $1 ; 47$ & 0.70 & 0.14 \\
\hline TMT-A (sec.) & 42.61 & 22.71 & 64.21 & 42.88 & 2.91 & $1 ; 47$ & $0.09 * *$ & 0.63 \\
\hline Declarative memory & & & & & 7.27 & $7 ; 41$ & $<0.01$ & \\
\hline digit span forward & 9.35 & 1.8 & 8.12 & 2.35 & 2.83 & $1 ; 47$ & 0.10 & 0.59 \\
\hline digit span backward & 6.21 & 2.36 & 5.65 & 2.04 & 0.50 & $1 ; 47$ & 0.48 & 0.25 \\
\hline block span forward & 8.50 & 2.04 & 9.99 & 2.02 & 3.96 & $1 ; 47$ & 0.05 & 0.73 \\
\hline block span backward & 7.16 & 2.19 & 5.68 & 2.41 & 4.06 & $1 ; 47$ & 0.05 & 0.64 \\
\hline VLMT total, trial 1-5 & 44.43 & 15.66 & 34.15 & 11.75 & 4.48 & $1 ; 47$ & 0.04 & 0.74 \\
\hline VLMT short delay & 2.54 & 2.57 & -0.65 & 1.46 & 14.28 & $1 ; 47$ & $<0.01$ & 1.53 \\
\hline VLMT long delay & 2.80 & 2.82 & 2.29 & 2.96 & 0.21 & $1 ; 47$ & 0.65 & 0.18 \\
\hline $\begin{array}{l}\text { Cognitive flexibility } \\
\text { TMT-B (sec.) }\end{array}$ & 106.78 & 50.52 & 139.29 & 82.79 & 1.63 & $1 ; 43$ & $0.21 *$ & 0.47 \\
\hline Neutral/ emotive memory & & & & & 0.01 & $2 ; 46$ & 0.99 & \\
\hline emotional memory & 9.39 & 3.53 & 9.52 & 3.06 & 0.01 & $1 ; 47$ & 0.92 & 0.04 \\
\hline neutral memory & 6.72 & 3.37 & 6.87 & 3.04 & 0.02 & $1 ; 47$ & 0.90 & 0.05 \\
\hline
\end{tabular}

ANCOVA $=$ Analysis of Covariance; MANCOVA = Multivariate Analysis of Covariance; $d f=$ degrees of freedom; TMT $=$ Trail Making Test; VLMT = Verbal Learning Memory Test; \# with IQ, years since having a psychiatric diagnosis, Young Mania Rating Scale scores, and current medication (antipsychotics; antidepressant \& mood stabilizer) as covariates; *without years since having a psychiatric diagnosis as covariate significant at $\mathrm{P}<0.05$; ** without YMRS scores or years since having a psychiatric diagnosis as covariates significant at $\mathrm{P}<0.05$. 
Table 3. Regression coefficients of the regression analyses in bipolar patients.

\begin{tabular}{|c|c|c|c|c|}
\hline Dependend variable & Predictor & Standardized Beta & $\mathbf{t}$ & $\mathbf{R}^{2}$ \\
\hline \multicolumn{5}{|c|}{ Attention and psychomotor speed } \\
\hline d2 speed (GZ) & Age & -0.60 & -3.74 & 0.36 \\
\hline $\mathrm{d} 2$ accurateness $(\mathrm{F} \%)$ & Age & 0.58 & 3.57 & 0.34 \\
\hline d2 concentration $(\mathrm{KL})$ & Age & $-0,72$ & -5.13 & 0.51 \\
\hline TMT-A (sec.) & Age & 0.64 & 4.15 & 0.41 \\
\hline \multicolumn{5}{|l|}{ Declarative memory } \\
\hline digit span forward & Age & -0.52 & -3.05 & 0.27 \\
\hline \multirow[t]{2}{*}{ block span backward } & $\begin{array}{l}\text { Antidepressants \& mood stabilizer } \\
\text { Age }\end{array}$ & -0.58 & $\begin{array}{l}-4.63 \\
-3.30\end{array}$ & 0.68 \\
\hline & & -0.41 & & \\
\hline VLMT total, trial 1-5 & Age & -0.74 & -5.46 & 0.54 \\
\hline VLMT long delay & Mood stabilizer only & -0.50 & -2.89 & 0.25 \\
\hline \multicolumn{5}{|l|}{ Cognitive flexibility } \\
\hline TMT-B (sec.) & Age & 0.73 & 5.35 & 0.53 \\
\hline
\end{tabular}

Only results with $\mathrm{p}<0.01$ are depicted.

Table 4. Regression coefficients of the regression analyses in schizoaffective patients.

\begin{tabular}{|c|c|c|c|c|}
\hline Dependend variable & Predictor & Standardized Beta & $\mathbf{t}$ & $\mathbf{R} 2$ \\
\hline $\begin{array}{l}\text { Attention and psychomotor speed } \\
\text { TMT-A (sec.) }\end{array}$ & Antidepressants \& Antipsychotics & 0.88 & 6.69 & 0.78 \\
\hline $\begin{array}{l}\text { Declarative memory } \\
\text { block span forward }\end{array}$ & Antidepressants \& mood stabilizer & -0.69 & -3.43 & 0.48 \\
\hline $\begin{array}{l}\text { Cognitive flexibility } \\
\text { TMT-B (sec.) }\end{array}$ & 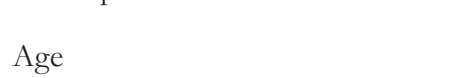 & 0.74 & 3.63 & 0.55 \\
\hline
\end{tabular}

Only results with $\mathrm{p}<0.01$ are depicted.

sample. Alcohol consumption was comparable between the two groups; only two bipolar patients exhibited mild alcohol abuse. Young Mania Rating Scale scores, years since having a psychiatric diagnosis, premorbid IQ and current medication (i.e. frequency of intake of antipsychotics or the combination of antidepressants and mood stabilizers) differed significantly between the two groups. Hence, these variables were included as covariates in the subsequent analyses.

\section{Neurocognitive Function}

As indicated in Table 2, significant group differences regarding attention and psychomotor speed (Pillai's trace $F=6.94 ; d f=4 ; 44 ; P<0.01)$ were found, even when controlled for possible confounding variables (i.e. years since having a psychiatric diagnosis, premorbid IQ, YMRS scores and current medication). The distinction between schizoaffective and bipolar patients accounted for $39 \%$ of the variance of the perfomance. Consecutively applied ANCOVA revealed that this result entirely arose from significant group differences with the accurateness of the $\mathrm{d} 2$ test $(F=12.37 ; d f=$ $1 ; 47 ; P<0.01)$. Schizoaffective patients also showed a trend towards a slower psychomotor speed in the TMT-A test when there have been years since having a psychiatric diagnosis $(F=6.42 ; d f=1 ; 52 ; P=0.01)$ or the YMRS scores $(F=4.49 ; d f=1 ; 49 ; P=0.04)$ were not used as covariates in the analysis (ANCOVA).

Similar findings resulted with regard to declarative memory. MANCOVA indicated significant group dif- ferences (Pillai's trace $F=6.55 ; d f=5 ; 43 ; P<0.01$ ) accounting for a total of $43 \%$ of the variance. This finding was mainly due to the significant lower performance of the patients with a schizoaffective disorder in the VLMT short delay test $(F=14.28$; $d f=1 ; 47$; $P<0.01)$. Furthermore, additional ANCOVA without the covariate of YMRS scores or without "years since having a psychiatric diagnosis" revealed a trend towards a smaller digit span in the group of schizoaffective patients $(F=4.68, d f=1 ; 52 ; P=0.04)$.

This notwithstanding, no significant group scores were found with regard to cognitive flexibility and neutral resp. emotive memory. Again, an ANCOVA with the covariate "years since having a psychiatric diagnosis" detected a trend towards less cognitive flexibility (TMT-B) in the group of schizoaffective patients $(F=6.93, d f=1 ; 47 ; P=0.01)$.

\section{Association of Demographic and Clinical VARiables on Cognitive FunCtioning}

By means of a stepwise regression analyses variables of potentially influencing cognitive functioning were assessed separately for schizoaffective and bipolar patients. The effect of age, gender, years since having a psychiatric diagnosis, hospitalisation rate, outpatient treatment, premorbid IQ, Young Mania Rating Scale scores and current medication were considered as variables.

In the group of bipolar patients, all neuropsychological tests of attention and psychomotor speed were 
best predicted by age, with a maximum of $51 \%$ of the variance explanation of $\mathrm{d} 2$ accurateness (cf. Table 3 ). Results of the declarative memory age as well as medication were important variables for the tests. The use of combined antidepressants and mood stabilizers together with the age accounted for $68 \%$ of the variance concerning the block span backward. The intake of mood stabilizers predicted best the results of VLMT long delay task, explaining $25 \%$ of the variance. The patients' age was one of the most relevant influencing variables in the TMT-B test.

In the group of schizoaffective patients, less models turned out to be significant. The TMT-A test was best predicted by current use of antidepressants and antipsychotics, explaining $78 \%$ of the variance (cf. Table 4). Patients on antidepressants and mood stabilizers performed worse in the block span forward task and the medication accounted for $48 \%$ of the variance. Finally, TMT-B, a test representing cognitive flexibility, was most influenced by age.

\section{Discussion}

The aim of this study was to evaluate neurocognitive functioning of patients with schizoaffective disorder (SAD) and Bipolar I disorder (BD) patients in remission. There have already been a few studies that have analysed the neuropsychological performance between these two diagnostic entities. However, these studies have not yet considered the extent of the differences in neuropsychological test results and significant data was used synonymously for the relevance of the testing results. For this reason $\mathrm{SAD}$ patients and $\mathrm{BD}$ patients were compared on various neurocognitive test dimensions and the effect sizes were computed. Additionally, the influence of demographic and clinical variables on neuropsychological performance was also analysed separately for each group. The samples differed with respect to psychopathology, premorbid intelligence, duration of illness and gender ratio with a higher proportion of females in the SAD group.

Overall, the data demonstrate that SAD patients have significantly more neuropsychological dysfunction and worse test results concerning the tasks regarding attention as well as declarative memory. An effect size of 1.18 in $\mathrm{d} 2$-accurateness resp. 1.53 in the VLMT short delay task, show large effects according to Cohen's classification [10]. In the MANCOVA the item "years since having a psychiatric diagnosis" proved to be an important covariate. Putative significant group differences in the TMT-B test vanished after the comparison was controlled for this variable. This is a remarkable result, taking into account that executive deficits in BD qualify as differential deficits, i.e. that they significantly exceed deficits in other cognitive domains [47]. Thus, regardless of the remarkable dissimilarity the deficits are comparable with regard to the severity. This is in accordance with previous studies investigating $\mathrm{SAD}$ with most marked deficits in executive functioning [5].

Our findings replicate the results found by Torrent et al. [48]. They also revealed significantly poorer performance in attention and memory of SAD patients compared to BD patients. Furthermore, in the present study TMT-A and TMT-B test results were significantly different in the ANOVA, but not in the MANCOVA. The putative differing results of Szoke et al. [45] turn out to be also in line with this lack of significance. Although the authors discuss similiar results of subjects with SZ and SAD in the TMT test, there were no significant differences between the groups at all apart from the comparison with the healthy control group. Additionally, they did not check demographic charateristics, though the SAD group had a significantly lower educational level than the $\mathrm{BD}$ group. Moreover, the fact that not all tests of declarative memory revealed significant group differences is consistent with the findings of Glahn et al. [16]. They found no significant difference in the digit span task between $\mathrm{SAD}$ and $\mathrm{BD}$, albeit they only report the $F$ test for bipolar subjects with psychotic features.

The calculated effect sizes indicate a remarkable magnitude of cognitive differences between SAD and BD. The effect sizes for attention $(d=1.18)$ and declarative memory $(\mathrm{d}=1.53)$ exceed the effect sizes between $\mathrm{SZ}$ and BD for the same neurocognitive domain $(\mathrm{d}=0.6$ resp. $\mathrm{d}=0.34$ ) (Seidman et al., 2002). Even the absolut dysfunction of sustained attention and verbal declarative memory in subjects with $\mathrm{BD}$ is less stronger $(\mathrm{d}=0.69$ resp. $\mathrm{d}=0.96$ compared with healthy controls). Similar effect sizes are reported by Schretlen et al. [42]. Even though there are no significant differences in all of the cognitive domains, a considerable disparity exists in specific cognitive functions. However, these studies did not use the same neuropsychological tests battery as in our study. Seidman et al. [43], for instance, used a dichotomic listening task to assess attentional deficits, whereas the declarative memory was assessed using the Wechsler Memory Scale.

A strength of our study was the careful controlling for the effects of covariates. In the present study the estimated premorbid IQ was lower in the SAD than in the BD sample. Similar findings were reported from Daban et al. (2006), who compared SZ with BD patients. Notably, the worse cognitive functioning of SAD patients persisted, even when controlling for the premorbid IQ $[4,36]$. It should be noted, that SAD patients had a longer history of diagnosed psychiatric disorder of around 10 years compared to the BD patients, what may be due to a worse clinical course of illness. In this study, we considered the age of onset of the disorder as a covariate.

Furthermore we did not distinguish between psychotic or affective subtypes of schizoaffective disorder, since we primarily included euthymic patients. Retrospectively, it was too vague to rely on the clinical report to differentiate in both of these subgroups.

SAD patients are commonly treated with psychopharmacological agents, e.g. antidepressants, antipsychotic medication or mood stabilizers to improve psychotic or affective symptoms [29]. Significant group differences concerning medication were obvious in our study and, consequently, we considered medication as a confounding variable in our statistical analysis.

Our results regarding the association of demographic and clinical variables on cognitive functioning 
are in line with the reports in the literature. Dittmann et al. [12] found that age and medication are relevant variables influencing all cognitive domains in $\mathrm{BD}$ patients. The negative impact of medication on cognitive performance revealed in our stepwise regression analyses is well in accordance with the study results of Donaldson et al. [13] and Savitz et al. [41]; for differing findings cf. Roiser et al. [39]. There is only sparse data in the literature about cognitive functioning in a separate group of SAD patients. Results for SAD patients are often merged with SZ patients. Recently, Elie et al. [14] reported that antipsychotic medication were associated with poorer cognitive functioning in such a combined group. Our findings convincingly showed a negative impact of medication on cognitive functioning. The variance was explained in up to $78 \%$ at maximum in the TMT-B test, what is remarkably a strong effect.

It is to consider that antipsychotic medication with an antidopaminergic profile (e.g. D2/D3 receptor anatgonism) has a strong negative influence on cognitive functioning and depression. In our sample we assumed these special mechanisms of antipsychotics not to be a main factor to explain the differences of cognitive functioning between both groups since SAD patients received no antipsychotic monotherapy, combined therapy did not differ statistically significant between both samples and the ratio of substances with an antidopaminergic profile was not different within the groups. We concluded the effects to be similar in both samples concering the antidopaminergic aspect of medication.

Hence, apart from the mentioned class effects our data did not allow further comments on the influence on cognition of distinct psychotropic compounds. Furthermore, cognitive impairment resulted particularly under medication with combined agents, e.g. concurrent intake of antidepressants, antipsychotics or mood stabilizers.

Finally, to refer to another aspect in the discusseion, Ferrier and Thompson [15] criticised the weakness of many studies not to control for residual affective symptoms. In our study we did not analyse the relevance of affective symptoms, also, because of two different psychometric test scales applied in each sample to evaluate depressive symptomatology (HAMD, MADRS). Nevertheless, the fact that neither HAMD in the BD group nor MADRS in the SAD group significantly correlated with the cognitive performance questions the hypothesis of a strong influence of affective symptoms on cognition in our study sample.

Our results may partly contribute to the discussion of the nosological role of SAD. Basically, there have been three hypotheses discussed in the literature regarding the classification of SAD in relation to SZ and affective disorder [29]. One of the hypotheses suggest that SAD is not a distinct entity at all. According to this hypothesis, the whole category is recommended to be abolished in favour of a broader concept of the other disorders [28]. The second model assumes that SAD only represents the intersection of schizophrenia and affective disorder with no features of its own. Malhi et al. [29] describes SAD to be "a point on a continuum of affective-psychotic psychopathology".
Finally, the third hypothesis ascribes SAD to be a distinct entity in the spectrum of psychotic disorder. Our data rather contradicts the assumption of SAD not to exist as an entity. Cognitive impairment is obviously more marked even when controlling the effects of covariates. Whether it is a severe form of $\mathrm{BD}$ in the spectrum of affective disorder or a separate entity still remains in discussion.

The main methodological shortcomings of our study are the small sample size and the lack of a healthy control group. The later is of importance as the premorbid IQ was lower in the SAD than in the BD sample. It is to consider that the sample of SAD patients were not completly remitted concerning depressive symptoms. Remission criteria for depression were evaluated using HAMD in the BD group and MADRS in the SAD sample. The use of MADRS in the SAD group resulted of the better item structure and test reliability of MADRS compared to HAMD, but was regretably not applied in the BD sample.

Furthermore, we did not separate between patients with and without a history of psychotic symptoms. Since our sample consisted of euthymic resp. (partially) remitted patients, we did not obtain valid data concerning the clinical history of psychotic symptoms during the lifetime, retrospectively. These limitations may contribute to blur the comparison of neuropsychological test results of SAD and BD patients. Glahn et al. [17] reported on worse results in the spatial delayed response task of $\mathrm{BD}$ patients with a lifetime history of psychotic features.

Additionally, future studies should consider the effect of medication in more detail. Due to the limited sample size only a group-wise analysis of psychotropic medication was calculated in our study, albeit marked effects on cognition may result under treatment with certain single compounds. A longitudinal study instead of a cross-sectional design may further contribute to more detailed results.

Acknowledgements: We thank all the participants who so generously gave their time and consent to participate in the research.

Disclosures: All authors have no competing interests.

\section{REFERENCES}

1. Albus M, Hubmann W, Wahlheim, Sobizack N, Franz U, Mohr F. Contrasts in neuropsychological test profile between patients with first-episode schizophrenia and firstepisode affective disorders. Acta Psychiatr Scand 1996; 94: 87-93

2. Baethge C. Long-term treatment of schizoaffective disorder: review and recommendations. Pharmacopsychiatry 2003; 36(2): 45-56

3. Beblo T. Neuropsychologie affektiver Störungen. In: Lautenbacher S, Gaugel S. Neuropsychologie psychischer Störungen. Springer, Berlin Heidelberg, 2004

4. Berrenttini WH. Are schizophrenic and bipolar disorders related? A review of family and molecular studies. Biol Psychiatry 2000; 48(6): 531-8

5. Bornstein RA, Nasrallah HA, Olson SC, Coffman JA, Torello M, Schwarzkopf SB. Neuropsychological deficit in schizophrenic subtypes: paranoid, nonparanoid, and schizoaffective subgroups. Psychiatry Res 1990; 31: 15-24 
6. Brickenkamp R. Test d2 Aufmerksamkeits-BelastungsTest, Handanweisung. 9. ed. Hogrefe: Göttingen Bern Toronto Seattle, 2002

7. Burdick KE, Goldberg JF, Harrow M, Faull RN, Malhotra AK. Neurocognition as a stable endophenotype in bipolar disorder and schizophrenia. J Nerv Ment Dis 2006; 194: 255-60

8. Calabrese P. Amnestische Syndrome, ein klinisch- neuropsychologischer Beitrag zur Differenzierung unterschiedlicher Gedächtnisstörungen. Lengerich: Pabst Science Publishers, 1998

9. Clark L, Iversen SD, Goodwin GM. Sustained attention deficit in bipolar disorder. Br J Psychiatry 2002; 180: 313-319

10. Cohen J. Statistical Power Analysis fort he Behavioral Sciences. Erlbaum: Hillsdale, NJ, 1988

11. Daban C, Martinz-Aran A, Torrent C, Tabarés-Seisdedos R, Balanzá-Martínez V, Salazar-Fraile J, Selva-Vera G, Vieta E. Specifity of cognitive deficits in bipolar disorder versus schizophrenia. A systematic review. Psychother Psychosom 2006; 75(2): 72-84

12. Dittmann S, Henning-Fast K, et al. Cognitive functioning in euthymic bipolar I and bipolar II patients. Bipolar Disord 2008; 10: 877-887

13. Donaldson S, Goldstein LH, Landau S, et al. The Maudsley Bipolar Disorder Project: the effect of medication, family history, and duration of illness on IQ and memory in bipolar I disorder. J Clin Psychiatry 2003; 64: 8693

14. Elie D, Poirier M, Chianetta J, et al. (2009) Cognitive effects of antipsychotic dosage and polypharmacy: a study with the BACS in patients with schizophrenia and schizoaffective disorder. J. Psychopharmacol 2009 [Epub ahead of print]

15. Ferrier IN, Thompson JM. Cognitive impairment in bipolar affective disorder: implications for the bipolar diathesis. Br J Psychiatry 2002; 180: 293-5

16. Glahn DC, Bearden CE, Cakir S, Barrett JA, Najt P, Serap Monkul E, Maples N, Velligan DI, Soares JC. Differential working memory impairment in bipolar disorder and schizophrenia: effectof of lifetime history of psychosis. Bipolar Disord 2006; 8: 117-123

17. Glahn DC, Barrett J, Bearden CE, et al. Dissociable mechanisms for memory impairment in bipolar disorder and schizophrenia. Psychol Med 2006; 36(8): 1085-1095

18. Goldberg TE, Gold JM, Greenberg R, Griffin S, Schulz SC, Pickar D, Kleinman JE, Weinberger DR. Contrasts between patients with affective disorders and patients with schizophrenia on a neuropsychological test battery. Am J Psychiatry 1993; 150: 1355-62

19. Goodwin F, Jamison K. Manic-Depressive Illness. New York: Oxford University Press, 1990

20. Green MF. Cognitive impairment and functional outcome in schizophrenia and bipolar disorder. J Clin Psychiatry $2006 ; 67,9: 3-8$

21. Härting C, Markowitsch HJ, Neufeld H, Calabrese $P$, Deisinger K, Kessler J. Wechsler Gedächtnis Test. Huber: Bern, 1997

22. Hawkins KA, Hoffman R, Quilan D, Rakfeld J, Docherty $\mathrm{N}$, Sledge W. Cognition, negative symptoms and diagnosis: a comparison of schizophrenic, bipolar and control samples. J Neuropsychiatry Clin Neurosci 1997; 9:81-89

23. Helmstaedter M, Lendt M, Lux S. VLMT - Verbaler Lern- und Merkfähigkeits-test, 1. ed. Beltz: Göttingen, 2002

24. Howell DC. Statistical methods for psychology (4th ed.). Belmont, CA: Duxbury Press, 1997

25. Krabbendam L, Arts B, van Os J, Aleman A. Cognitive functioning in patients with schizophrenia and bipolar disorder: a qunatitative review. Schizophr Res 2005; 80: 137-149
26. Lehrl S. Mehrfachwahl-Wortschatz-Intelligenztest, MWTB. Balingen: Spitta, 5. ed., 2005

27. Lezak MD. Neuropsychological Assessment (3rd ed.). New York: Oxford University Press, 1995

28. Maier W. Do schizoaffective disorders exist at all? Acta Psychatr Scand 2006; 13: 369-371

29. Malhi GS, Green M, Fagiolini A, Peselow ED, Kumari V. Schizoaffective disorder: diagnostic issues and future recommendations. Bipolar Disord 2008; 10: 215-230

30. Marneos A, Goodwin FK. Mixed states, rapid cycling and atypical bipolar disorder. Cambridge: University Press, 2004

31. Martinez-Aran A, Vieta E, Torrent C, et al. Functional outcome in bipolar disorder: the role of clinial and cognitive factors. Bipolar Disord 2007; 9:103-113

32. Martinez-Aran A, Vieta E, Colom F, et al. Cognitive impairment in euthymic bipolar patients: implications for clinical and functional outcome. Bipolar Disorder 2004; 6: 224-232

33. Martinez-Arán A, Vieta E, Colom F, Torrent C, Reinares M, Goikolea JM, Benabarre A, Comes M, SánchezMoreno J. Do cognitive complaints in euthymic bipolar patients reflect objective cognitive impairment? Psychother Psychosom 2005; 74: 295-302

34. McIntosh AM, Harrison LK, Forrester K, Lawrie SM, Johnstone EC. Neuropsychological impairments in people with schizophrenia or bipolar disorder and their unaffected relatives. Br J Psychiatry 2005; 186: 378-85

35. Mitrushina MN, Boone KL, D'Elia L. Handbook of normative data for neuropsychological assessment. New York: Oxford University Press, 1999

36. Nardi AE, Nascimento I, Freire RC, et al. Demographic and clinical features of schizoaffective (schizobipolar) disorder - a 5-year retrospective study. Support for a bipolar spectrum disorder. J Affect Disord 2005; 89: 201206

37. Reitan RM, Wolfson D. The Halstead-Reitan Neuropsychological Test Battery: Therapy and clinical interpretation. Tucson, AZ: Neuropsychologial Press, 1985

38. Robinson L, Thompson JM, Gallagher P, et al. A metaanalysis of cognitive deficits in euthymic patients with bipolar disorder. J Affect Disord 2006; 93: 105-115

39. Roiser JP, Cannon DM, Gandhi SK, Taylor Tavares J, Erickson K, Wood S, Klaver JM, Clark L, Zarate CA Jr, Sahakian BJ, Drevets WC. Hot and cold cognition in unmedicated depressed subjects with bipolar disorder. Bipolar Disord 2009; 11: 178-89

40. Rubinsztein JS, Michael A, Paykel ES, Sahakian BJ. Cognitive impairment in remission in bipolar affective disorder. Psychol Med 2000; 30: 1025-1036

41. Savitz JB, van der Merwe L, Stein DJ, Solms M, Ramesar RS. Neuropsychological task performance in bipolar spectrum illness: genetics, alcohol abuse, medication and childhood trauma. Bipolar Disord 2008; 10: 479-94

42. Schretlen DJ, Cascella NG, Meyer SM, et al. Neuropsychological functioning in bipolar disorder and schizophrenia. Biol Psychiatry 2007; 62:179-86

43. Seidman LJ, Kremen WS, Koren D, Faraone SV, Goldstein JM, Tsuang MT. A comparative profile analysis of neuropsychological functioning in patients with schizophrenia and bipolar psychoses. Schizophr Res 2002; 53: 31-44

44. SPSS Inc. SPSS for Windows Version 14.0., Chicago, 2006

45. Szoke, A, Meary, A, Trandafir A, et al. Executive deficits in psychotic and bipolar disorders - Implicationen for our underständing of schizoaffective disorder. Eur Psychiatry 2007; 27: 20-25

46. Tam WC, Sewell K, Deng H. Information processing in schizophrenia and bipolar disorder: a discriminant analysis. J Nerv Ment Dis 1998; 186:597-603 
47. Thompson JM, Gray JM, Crawford JR, Hughes JH, Young AH, Ferrier IN. Differential deficit in executive control in euthymic bipolar disorder. J Abnorm Psychol 2009; 118:146-60

48. Torrent C, Martinez- Aran A, et al. Cognitive impairment in schizoaffective disorder : a comparison with non-psychotic bipolar and healthy subjects. Acta Psychiatr Scand 2007; 116: 453-460

49. Wechsler D. Wechsler Adult Intelligence Scale. Cleveland, Ohio: The Psychological Corporation, 1955

50. Zalla, Joyce C, Szöke A, et al. Executive dysfunctions as potential markers of familial vulnerability to bipolar disorder and schizophrenia. Psychiatr Res 2004; 121: 207-17

51. Zubieta J, Huguelet P, O’Neil RL, Giordani BJ. Cognitive function in euthymic bipolar I disorder. Psychiatr Res 2001; 102: 9-20
Received: September 23, 2009 / Accepted: November 24, 2009

Address for correspondence:

Hans-Jörg Assion, MD

Dept. of Psychiatry and Psychotherapy gpz Detmold

Schlabrendorffstr. 2-6

32756 Detmold

Germany

Phone: +49-5231-45850-900

Fax: +49-5231-45850-905

Email: assion-hj@versanet.de 